\title{
Advanced Signal Processing for Cognitive Radio Networks
}

\author{
Ying-Chang Liang, ${ }^{1}$ Xiaodong Wang, ${ }^{2}$ Yonghong Zeng, ${ }^{1}$ Jinho Choi, ${ }^{3}$ \\ Rui Zhang, ${ }^{1}$ and Marco Luise ${ }^{4}$ \\ ${ }^{1}$ Institute for Infocomm Research, A*STAR, Singapore 138632 \\ ${ }^{2}$ Department of Electrical Engineering, Columbia University, New York, NY 10027, USA \\ ${ }^{3}$ School of Engineering, Swansea University, Singleton Park, Swansea, SA2 8PP, UK \\ ${ }^{4}$ Dipartimento di Ingegneria dell'Informazione, Università degli Studi di Pisa, 56100 Pisa, Italy \\ Correspondence should be addressed to Ying-Chang Liang, ycliang@i2r.a-star.edu.sg
}

Received 20 December 2009; Accepted 20 December 2009

Copyright ( 2010 Ying-Chang Liang et al. This is an open access article distributed under the Creative Commons Attribution License, which permits unrestricted use, distribution, and reproduction in any medium, provided the original work is properly cited.

Cognitive radio is the enabling technology for dynamic spectrum access, which may potentially mitigate the radio spectrum scarcity problem encountered in many countries. Thus, cognitive radio is widely regarded as one of the most promising technologies for the future wireless communications. For instance, the Federal Communications Commission (FCC) of the US approved in November 2008 the use of mobile devices in unused TV bands (TV white spaces), and many governments worldwide have also moved to support this new spectrum sharing model. This has been recently accompanied by a significant upsurge in academic research and application initiatives, especially in unlocking the potential in the "white space" of TV bands for various broadband wireless services.

However, cognitive wireless networking is still in the early stages of research and development. To achieve fully "cognitive" transmission over a wireless network, there are still tremendous technical, economical, and regulatory challenges. Signal processing plays a major role in designing cognitive wireless networks, especially in spectrum sensing for identifying spectrum opportunities and cognitive spectrum access design for exploiting the identified spectrum opportunities. The aim of this special issue is just to provide a collection of high-quality research papers in advanced signal processing for cognitive radio networks addressing various fundamental as well as system design problems.

For this special issue, we have received 18 paper submissions altogether. After careful review over all the submissions, 7 papers have been accepted for publications.
The first paper is an overview paper, entitled "A review on spectrum sensing techniques for cognitive radio: challenges and solutions" and co-authored by Zeng et al. This paper provides a systematic overview on the spectrum sensing techniques for cognitive radio, including conventional as well as recently developed sensing schemes. The authors also list down the challenges of spectrum sensing design due to practical considerations and requirements and compare the applicability of various sensing schemes in different scenarios.

The second paper, "Securing collaborative spectrum sensing against Untrustworthy secondary users in cognitive radio networks" by Wang et al., addresses the problem of collaborative spectrum sensing by detecting the untrustworthy secondary users which may report false sensing results purposely to the fusion center.

The third paper is entitled "Spectral correlation of multicarrier modulated signals and its application for signal detection" and co-authored by Zhang et al. It investigates the cyclostationarity characteristics for two kinds of multicarrier modulated (MCM) signals, including conventional OFDM and filter bank based multicarrier (FBMC) signals. Cyclostationary Signatures are also artificially embedded into MCM signal and a low-complexity signature detector ispresented for detecting MCM signal.

The fourth paper is co-authored by Kocak et al., and is entitled "Time delay estimation in dispersed spectrum cognitive radio systems." It addresses the problem of time delay estimation in cognitive radio systems facilitating opportunistic use of spectral resources. A two-step approach 
is proposed to obtain accurate time delay estimates of signals that occupy multiple dispersed bands simultaneously, with significantly lower computational complexity than the optimal maximum likelihood (ML) estimator.

In the fifth paper entitled "Uplink user signal separation for OFDMA-based cognitive radios," Sahin et al. study the problem of separation of primary user signals in the uplink of orthogonal frequency division multiple access (OFDMA) based cognitive radio systems, for the purpose of increasing the spectrum awareness of the cognitive radios.

The sixth paper is co-authored by Xiao et al., and entitled "A unified approach to optimal OSA under collision probability constraint in cognitive radio systems". The authors study the optimal opportunistic spectrum access problem and formulate it as an optimization problem that the secondary user maximizes spectrum hole utilization under the constraint of collision tolerable level of the primary users.

In the seventh paper, "Optimal policy of Cross-layer design for channel access and transmission rate adaptation in cognitive radio networks," He et al. investigate the cross-layer design of joint channel access and transmission rate adaptation in cognitive radio networks with multiple channels for both centralized and decentralized cases. The design target is to maximize the throughput of cognitive radio network under transmitting power constraint by taking spectrum sensing errors into account for both centralized and decentralized networks.

We would like to thank all authors for their contributions to this special issue, the reviewers for their effort and time spent in reviewing the papers, and finally the editor Phillip Regalia for his support during the whole process.

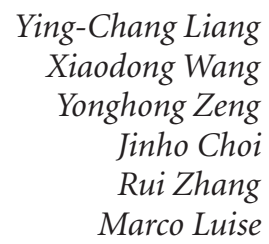

